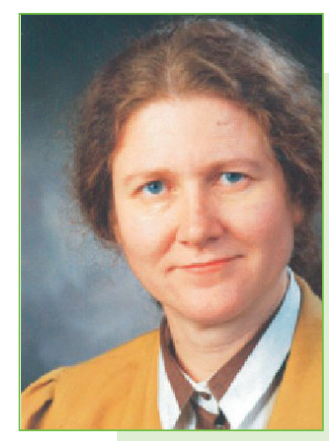

\title{
Praktische Aspekte der Phytotherapie
}

\section{Editorial}

Die seit dem 1. 7. 2006 in Deutschland in Kraft getretene Zuzahlungsbefreiung für Arzneimittel, die 30\% günstiger sind als der von den Kassen festgelegte Höchstpreis, greift offenbar sehr gut. Die Zuzahlung von fünf bis zehn Euro ist bis Mitte August schon für 2637 Arzneimittel weggefallen. Weitere Arzneimittel werden bis zum 1.11.2006 folgen. Ob sich daraus für die Bereitschaft der Patienten, Phytopharmaka zu kaufen und einzunehmen, Konsequenzen ergeben, bleibt abzuwarten. Betroffen werden wohl vor allem diejenigen Phytopharmaka sein, die bei Erkrankungen eingesetzt werden, die auch mit erstattungsfähigen, chemisch definierten Arzneimitteln behandelt werden können, wie z.B. die pflanzlichen Prostatamittel. Es ist gut vorstellbar, dass noch mehr Patienten aus finanziellen Überlegungen heraus dem chemisch definierten Prostatamittel den Vorzug geben werden.

Was lässt die Patienten überhaupt zu pflanzlichen Arzneimitteln greifen? Bekannt ist, das pflanzliche Arzneimittel vor allem von Frauen, und zwar insbesondere für chronische, unheilbare oder immer wieder auftretende Krankheiten eingesetzt werden. Finanzielle Überlegungen scheinen jedoch nicht im Vordergrund $\mathrm{zu}$ stehen. International betrachtet, steigt der Gebrauch von pflanzlichen Arzneimitteln weiter an, weil über sie immer häufiger in den Medien berichtet wird, und weil die meisten Patienten glauben, dass pflanzliche Arzneimittel infolge ihres natürlichen Ursprungs sicherer sind als konventionelle Arzneimittel. Dies ist offenbar auch die Ursache dafür, dass der Gebrauch von Phytopharmaka oft dem behandelnden Arzt nicht mitgeteilt wird. Während aber in Deutschland Phytopharmaka dem Arzneimittelgesetz unterliegen und ihre Unbedenklichkeit nachgewiesen sein muss, wird z. B. die amerikanische Food and Drug Administration erst aktiv, wenn Berichte über unerwünschten Wirkungen von bereits im Markt befindlichen Produkten erscheinen. Dass diese Berichte seit Jahren von einer zweifelhaften Qualität sind, auch wenn sie in renommierten Fachblättern erschienen sind, erschwert den Beurteilungsprozess nicht nur für die Fachleute.

Über die Motivation zum Einsatz pflanzlicher Arzneimittel habe ich kürzlich eine interessante Untersuchung aus Italien gefunden (Zaffani S, Cuzzolin L, Benoni G: Herbal products: behaviors and beliefs among Italian women. Pharmacoepidemiology and Drug Safety 2006; 15: 354-9). In dieser Studie wurden 1063 Frauen (Alter zwischen 30 und 50 Jahre), die eine allgemeinmedizinische Ambulanz an der
Universität Verona aufsuchten, anonym mit einem Fragebogen befragt. In den vergangenen 12 Monaten hatten 491 Frauen mindestens ein pflanzliches Arzneimittel eingenommen, davon 31,6\% häufiger. 35,2\% von den Anwenderinnen hatten auch an ihre Kinder Phytopharmaka verabreicht. Bei den Nichtanwenderinnen bestand allgemein Aufgeschlossenheit gegenüber einem zukünftigen Gebrauch. Je höher der Bildungsgrad war, desto häufiger wurden Phytopharmaka verwendet. Hauptgründe für die Einnahme waren häufige Erkältungen und gastrointestinale oder respiratorische Probleme. 9,6\% der Anwenderinnen glaubten, unerwünschte Wirkungen im $\mathrm{Zu}$ sammenhang mit der Einnahme verspürt zu haben, von diesen sprach aber nur ein Drittel darüber mit ihrem Arzt. Über 50\% kauften die Produkte in Apotheken, fast $75 \%$ wurden bei ihrem Kauf nicht über das Produkt aufgeklärt. 62\% informierten ihren Arzt nicht über den Gebrauch, offenbar weil sie die Mittel für harmlos und den Arzt als inkompetent oder nicht aufgeschlossen einschätzten. Der Hauptgrund für die Einnahme war die Vorstellung, dass die pflanzlichen Medikamente wegen ihrer natürlichen Herkunft sicherer wären.

Welche Konsequenzen sollten daraus gezogen werden? Am wichtigsten erscheint mir, sachlich, unabhängig und fair zu informieren, damit emotionale Argumente durch korrekte Sachargumente ersetzt werden. Das Thema "Arzneimittelsicherheit» spielt gegenwärtig in der wissenschaftlichen und legislativen Diskussion eine größere Rolle als die Belege der Wirksamkeit. Daraus könnte tatsächlich noch die Aufdeckung von Risiken bei einigen pflanzlichen Arzneimitteln resultieren. Die meisten Kasuistiken sind allerdings diesbezüglich nicht zielführend. Hier ist dringend eine höhere Qualität zu fordern.

Ebenso wichtig scheint es zu sein, dass die Ärzte und Apotheker bereits während ihrer Ausbildung ausreichende und wissenschaftlich fundierte Informationen über Phytotherapie erhalten, um ihren Patienten bei Bedarf korrekte Informationen über ihre Qualität, Wirksamkeit und Unbedenklichkeit geben zu können. Gegenwärtig sind die Rahmenbedingungen dafür allerdings an den meisten deutschen Universitäten, und nicht nur dort, relativ ungünstig. Nur gemeinsame Anstrengungen aller Akteure können die gegenwärtig bestehenden massiven Probleme lösen. Je eher wir beginnen, desto besser.

Karin Kraft 